\title{
Machine learning can differentiate venom toxins from other proteins having non-toxic physiological functions
}

\author{
Ranko Gacesa $^{1}$, David J Barlow ${ }^{1}$ ， Paul F Long ${ }^{\text {Corresp. 1, 2, 3,4 }}$ \\ 1 Institute of Pharmaceutical Science, King's College London, London, United Kingdom \\ 2 Department of Chemistry, King's College London, London, United Kingdom \\ 3 Brazil Institute, King's College London, London, United Kingdom \\ 4 Faculdade de Ciências Farmacêuticas, Universidade de São Paulo, São Paulo, Brazil \\ Corresponding Author: Paul F Long \\ Email address: paul.long@kcl.ac.uk
}

Ascribing function to sequence in the absence of biological data is an on-going challenge in bioinformatics. Differentiating the toxins of venomous animals from homologues having other physiological functions is particularly problematic as there are no universally accepted methods by which to attribute toxin function using sequence data alone. Bioinformatics tools that do exist are difficult to implement for researchers with little bioinformatics training. Here we announce a machine learning tool called 'ToxClassifier' that enables simple and consistent discrimination of toxins from non-toxin sequences with $>99 \%$ accuracy and compare it to commonly used toxin annotation methods.

'ToxClassifer' also reports the best-hit annotation allowing placement of a toxin into the most appropriate toxin protein family, or relates it to a non-toxic protein having the closest homology, giving enhanced curation of existing biological databases and new venomics projects. 'ToxClassifier' is available for free, either to download ( https://github.com/rgacesa/ToxClassifier) or to use on a web-based server ( http://bioserv7.bioinfo.pbf.hr/ToxClassifier/). 
1 Machine learning can differentiate venom toxins from other proteins having non-toxic

2 physiological functions

3

4 Ranko Gacesa ${ }^{1}$, David J. Barlow ${ }^{1,2}$, Paul F. Long ${ }^{1-4 *}$

5

6 'Institute of Pharmaceutical Science, King's College London, 150 Stamford Street, London SE1

7 9NH, United Kingdom.

8 2Department of Chemistry \& ${ }^{3}$ Brazil Institute, King's College London, Strand, London WC2R

9 2LS, United Kingdom.

$10{ }^{4}$ Faculdade de Ciências Farmacêuticas, Universidade de São Paulo, Av. Prof. Lineu Prestes, 11 580, B16, 05508-000 São Paulo, SP, Brazil.

12

13 *Corresponding author:

14 Prof Paul F. Long

15 email: paul.long@kcl.ac.uk

16 tel/fax: $0044(0) 2074844842$

17 


\section{Abstract}

Ascribing function to sequence in the absence of biological data is an on-going challenge

20 in bioinformatics. Differentiating the toxins of venomous animals from homologues having other

21 physiological functions is particularly problematic as there are no universally accepted methods

22 by which to attribute toxin function using sequence data alone. Bioinformatics tools that do exist

23 are difficult to implement for researchers with little bioinformatics training. Here we announce a

24 machine learning tool called 'ToxClassifier' that enables simple and consistent discrimination of

25 toxins from non-toxin sequences with $>99 \%$ accuracy and compare it to commonly used toxin

26 annotation methods. 'ToxClassifer' also reports the best-hit annotation allowing placement of a

27 toxin into the most appropriate toxin protein family, or relates it to a non-toxic protein having the

28 closest homology, giving enhanced curation of existing biological databases and new venomics

29 projects. 'ToxClassifier' is available for free, either to

30 download (https://github.com/rgacesa/ToxClassifier) or to use on a web-based

31 server (http://bioserv7.bioinfo.pbf.hr/ToxClassifier/). 


\section{Introduction}

Falling costs of tandem mass spectrometry for shotgun proteomics have made generating vast amounts of protein sequence data increasingly affordable, yet the gap between obtaining these sequences and then assigning biological function to them continues to widen (Bromberg et al. 2009). Often, most sequences are deposited into protein databases with little, if any, accompanying experimental data from which biological functions can be inferred. Customarily, biological function is deduced indirectly by comparing amino acid sequence similarity to other proteins in large databases to calculate a ranking of proteins with respect to the query sequence. Using simple pair-wise comparisons as a sequence searching procedure, the BLAST suite of programs (for example, BLASTp) was first of its kind and has gained almost unprecedented acceptance among scientists (Neumann et al. 2014). Variations of the BLAST algorithm (for instance, PSI-BLAST (Altschul et al. 1997)) and development of probabilistic models (such as hidden Markov models, HMMs (Krogh et al. 1994)) use multiple sequence alignments to detect conserved sequences (also referred to as motifs). Use of these models enables detection of remote homology between proteins seemingly unrelated when analysed by pairwise alignment alone (Krogh et al. 1994). Conversely, development of accurate algorithms and fast software tools that can automatically identify critical amino acid residues responsible for differences in protein function amongst sequences having exceptionally high sequence similarity remains a challenging problem for bioinformatics. In a post-genomic era, the toxins of venomous animals are an emerging paradigm.

Animal venoms comprise predominantly toxic peptides and proteins. Duplication of genes that formerly encoded peptides and proteins having non-toxic physiological functions is one of the foremost evolutionary drivers that gives rise to the enormous functional diversity seen 
56 in animal venom toxins (Fry 2005; Chang \& Duda 2012). However, evidence is ambiguous as to

57 whether these genes were expressed in multiple body tissues, with the duplicate copy then

58 recruited into venom glands with subsequent neo-functionalisation to develop toxicity, or if there

59 was duplication with ensuing sub-functionalisation of genes encoding pre-existing but non-toxic

60 venom gland proteins (Hargreaves et al. 2014). Examples of both mechanisms have been

61 demonstrated in different venomous animals (Reyes-Velasco et al. 2015; Vonk et al. 2013;

62 Junqueira-de-Azevedo et al. 2015). Nonetheless, many toxin proteins that constitute venoms

63 share a remarkable similarity to other proteins with non-toxic physiological functions, and

64 deciphering sequence data to disentangle these functions is not a trivial task (Kaas \& Craik 65 2015).

66 Previous proteomic data from our laboratory and subsequent results of others realised an 67 astonishingly high sequence similarity between cnidarian (jellyfish, coral, sea anemones etc.) 68 toxins and those of other higher venomous animals (Weston et al. 2012; Weston et al. 2013; Li et 69 al. 2012; Li et al. 2014). This suggested to us that a small number of sequences, when occurring 70 in combination, might explain this similarity and prompted the search for toxin-specific motifs 71 (Starcevic \& Long 2013). An unsupervised procedure was developed that resulted in the 72 identification of motifs we called 'tox-bits', and which could describe most toxins as 73 combinations of between 2-3 'tox-bits' (Starcevic et al. 2015). The 'tox-bits' are defined as 74 HMM-profiles and were found to be superior at differentiating toxin from non-toxin sequences, 75 when compared against standard BLAST or HMM based methods (Starcevic et al. 2015). 76 However, implementation of 'tox-bits' HMM profiles is not straightforward for scientists with

77 little or no bioinformatics experience. Hence, in this paper we introduce and make freely 78 available an easy-to-use machine learning tool called the 'ToxClassifier' that employs 'tox-bits' 
79 HMM profiles and other standard classifier tools running in parallel to distinguish toxins from

80 their non-toxic homologues.

81

82 


\section{Methods}

84 Datasets

85 Data for training and testing of machine learning classifiers used in ToxClassifier ensemble was 86 obtained from UniProtKB database (Bateman et al. 2015), according to the following 87 methodology:

88 1) 'Positive' dataset representing well annotated putative animal toxins was downloaded from 89 UniProtKB/SwissProt-ToxProt (Jungo et al. 2012). Database was searched for animal toxins 90 and venoms, using search query: taxonomy:"Metazoa [33208]" (keyword:toxin OR 91 annotation:(type:"tissue specificity" venom)). All duplicate entries with identical sequence or 92 sequence identifier were removed, resulting in 8,093 sequences.

93 2) 'Easy' negative dataset representative of physiological proteins was obtained by random 94 sampling of 50,000 sequences in UniProtKB/SwissProt database (Bateman et al. 2015). All 95 entries with duplicate sequence identifier or protein sequence were removed, as were all 96 entries also occuring in Positive dataset; final dataset included 47,144 protein sequences.

97 3) 'Moderate difficulty' negative dataset was designed to match highly curated toxin-like 98 proteins with physiological function; it was created by BLASTp searching 99 UniProtKB/SwissProt database with Positive dataset, with e-value cutoff of 1.0e-10.

100 Resulting BLASTp hits were collected and all duplicates (with identical sequence or 101 sequence identifier), and all sequences also present in Positive dataset or Easy dataset were 102 removed, resulting in 8,034 proteins.

103 4) 'Hard' negative dataset was constructed from TrEMBL database (Bairoch \& Apweiler 2000)

104 instead of Swiss-Prot. As with Moderate dataset, it was created from results of BLASTp 105 using Positive dataset as query and TrEMBL as target database. Duplicates and sequences 
106 also occurring in Positive, Easy or Moderate datasets were removed for total of 7,403

107 sequences.

108 All datasets are available for download at https://github.com/rgacesa/ToxClassifier/datasets.

109 Machine learning classifiers, training and testing

110 Models describing protein sequences were constructed as follows:

111 1) Single Amino acid frequency model (OF): model uses length of sequence and frequency of 112 each amino acid as input features.

113 2) Amino acid dimer frequency model (BIF): model uses length of sequence, frequency of each 114 amino acid and of each amino acid 2-mer.

115 3) Naive tox-bits model (NTB): input features for this model are the number of 'tox-bits' for 116 each 'tox-bits' HMM listed in the 'tox-bits' database (Starcevic et al. 2015).

117 4) Scored 'tox-bits' model (STB): STB is a modification of the NTB model, with HMM bit118 scores replacing the number of 'tox-bits' in each 'tox-bit' HMM model.

119 5) Tri-Blast Simple (TBS) model: TBS uses BLASTp searches against positive 120 (UniProtKB/SwissProt-ToxProt) and two negative control databases (close non-toxins from 121 UniProtKB/SwissProt and non-toxins from TrEMBL); features include bit-score, query 122 length, subject length, query/subject length ratio, query coverage, percentage of identity, 123 percentage of positive matches; features also include amino-acid frequencies. Scores are 124 computed from the 'best-hit' in each database, with a BLAST e-value of 1.0e-10.

125 6) Tri-Blast Enhanced $A$ (TBEa) model: TBEa model is an expanded variant of TBS, with 126 amino dimer frequencies included in the model. 
127 7) Tri-Blast Enhanced $B$ (TBEb): model is a variation of TBEa, trained on $80 \%$ of the input

128 dataset and with a BLAST e-value cut-off value of $1.0 \mathrm{e}+3$ for the detection of similar toxin

129 or non-toxic sequences.

130 Feature extraction and vectorisation was implemented using the Python 2.7

131 (https://www.python.org/download/releases/2.7/) scripting language, NCBI BLAST+ version

132 2.2.31 (Camacho et al. 2009), HMMER 3.1b1 (Eddy 2011), the 'tox-bit' HMM profile database

133 (Starcevic et al. 2015) and a set of custom BLAST databases based on UniProtKB/SwissProt-

134 ToxProt, UniProtKB/SwissProt and TrEMBL databases. Vectorization scripts, vectorised data

135 sets and trained models are available for download at https://github.com/rgacesa/ToxClassifier.

136 Support Vector Machine (SVM), Gradient Boosted Machine (GBM) and Generalised Linear

137 Model (GLM) classifiers were trained for each of the models; Classifiers were implemented

138 using the R-programming language, Caret package (http://topepo.github.io/caret/index.html).

139 The training set for each dataset was selected by random sampling of $75 \%$ of the sequences, and

140 combined training sets were used to train the classifiers. Input features were 0-centered and

141 scaled by standard deviation and training was performed with 10 internal bootstraps. Learning

142 curves were constructed for each classifier to evaluate training efficiency and potential

143 overtraining by plotting performance versus number of sequences in training set.

144 Classifiers were tested using those sequences from Positive, Easy, Moderate and Hard datasets

145 not used in training. Performance was evaluated on each of the datasets and on the summary

146 dataset. The following performance measurements were calculated:

147 1) Number of true positives (TP): Number of toxic sequences in dataset correctly predicted as 148 toxins; sequence was considered a toxin if listed in UniProtKB/ToxProt database. 
149 2) Number of true negatives (TN): Number of non-toxic sequences in dataset which are

150 correctly predicted as non-toxic (not in UniProtKB/ToxProt database).

151 3) Number of false positive (FP): Number of non-toxic sequences in dataset incorrectly $152 \quad$ classified as toxins.

153 4) Number of false negatives (FN): Number of toxic sequences in dataset incorrectly classified 154 as non-toxic.

155 5) Accuracy (ACC): Accuracy is calculated as proportion of sequences correctly classified as 156 toxins or non-toxins; $\mathrm{ACC}=(\mathrm{TP}+\mathrm{TN}) /(\mathrm{TP}+\mathrm{TN}+\mathrm{FP}+\mathrm{FN})$.

157 6) Specificity (SPEC): Also called true negative rate, specificity is proportion of correctly 158 predicted non-toxins (true negatives); $\mathrm{SPEC}=\mathrm{TN} /(\mathrm{TN}+\mathrm{FP})$.

159 7) Sensitivity (SENS): Also called true positive rate or recall, sensitivity is proportion of 160 correctly predicted toxins (true positives); $\mathrm{SENS}=\mathrm{TP} /(\mathrm{TP}+\mathrm{FN})$.

161 8) Balanced Accuracy (B.ACC): Balanced accuracy is a mean value of specificity and 162 sensitivity; B.ACC $=($ SPEC + SENS $) / 2$.

163 9) Negative predictive value (NPV): Proportion of negatives that are true negatives. NPV $=$ TN $164 \quad /(\mathrm{TN}+\mathrm{FN})$.

165 10) Positive predictive value (PPV): Also called Precision, PPV measures proportion of positives 166 which are true positives; $\mathrm{PPV}=\mathrm{TP} /(\mathrm{TP}+\mathrm{FP})$.

167 11) F-Score (F1): F-score is harmonic mean of precision and sensitivity and represents weighted 168 average of precision and recall. It is calculated as $\mathrm{F} 1=2 * \mathrm{TP} /(2 * \mathrm{TP}+\mathrm{FP}+\mathrm{FN})$.

169 12) Matthews' correlation coefficient (MCC): The MCC value (also known as phi-coefficient) is 170 a measure of correlation between observed and predicted. It is considered balanced measure 
171 even for classes with different amounts of positive and negative values; $\mathrm{MCC}=$

$172 \frac{T P * T N-F P * F N}{\sqrt{(T P+F P) *(T P+F N) *(T N+F P) *(T N+F N)}}$ (Matthews 1975; Powers 2011).

\section{Conventional annotation models}

174 Annotation models simulating manual annotation were constructed based on BLAST and 175 HMMER, as follows:

176 1) Naive-BLAST: Annotation method is based on the assumption that a sequence is a toxin if a

177 BLAST search against the UniProtKB/SwissProt-ToxProt dataset returns a positive hit at a 178 certain e-value (usually between 1.0e-20 and 1.0e-5). This approach and its variations are 179 commonly encountered in the literature (Sher \& Zlotkin 2009; Schwartz et al. 2015; Liu et al. 180 2015; Whittington et al. 2010).

181 2) OneBLAST: This approach classifies a sequence by a single BLAST search against 182 databases including UniProtKB/SwissProt-ToxProt and other 'toxin-like' but non-toxic 183 sequences extracted from UniProtKB/SwissProt and TrEMBL databases (combination of 184 Positive, Moderate and Hard datasets). A sequence is classified as a putative toxin if the 185 highest scoring BLAST hit at a selected e-value is from UniProtKB/SwissProt-ToxProt; it is 186 classified as non-toxic if top BLAST hit is not from UniProtKB/SwissProt-ToxProt, or if all 187 BLAST hits are above the selected e-value.

188 3) TriBLAST: Annotation is performed by a variation of OneBLAST, using separate BLAST 189 searches against UniProtKB/SwissProt-ToxProt and two non-toxin databases (for the 190 Moderate and Hard datasets); a sequence is classified as a toxin if the BLAST search against

191 UniProtKB/SwissProt-ToxProt returns a hit below a certain e-value and with a higher score 192 than the searches against the other databases. Variations of this method are commonly used 193 in toxin annotation (Gacesa et al. 2015; Rachamim et al. 2014). 
194 4) hmmerToxBits: This method is a variation of the naive-BLAST, using HMMER package

195 Hmmsearch instead of BLAST, and the database of 'tox-bits' HMM models as the target

196 database. A sequence is classified as a toxin if one or more HMMs can be detected within a

197 certain e-value cut-off.

198 5) hmmerVenom: Modification of hmmerToxBits, the method uses HMM profiles extracted by

199 'venom' and 'toxin' text search of the Pfam database instead of 'tox-bit' HMMs.

200 6) twinHmmerPfam: A HMMER based variant of TriBLAST approach, this method performs 201 hmmsearches against two HMM databases (toxin/positive HMMs and negative control) and 202 compares bitscores. Sequence is annotated as a toxin if bitscore for toxin HMM database is 203 higher. TwinHmmerPfam toxin HMMs were extracted from Pfam by keyword search for 204 'toxin' and 'venom', while negative control database is comprised from remainder of Pfam.

205 7) twinHmmerToxBits: A variation of twinHmmerPfam method, method compares hmmsearch 206 against toxin HMMs and negative control. For this model, positive database is composed 207 from 'tox-bit' HMMs derived from Tox-Prot toxins (Starcevic et al. 2015), while negative 208 control is Pfam database from which HMMs containing 'toxin' or 'venom' keywords were 209 removed.

210 BLAST databases for Naive-BLAST, OneBLAST and TriBLAST were constructed from $75 \%$ 211 randomly sampled sequences in Positive, Easy, Moderate and Hard and performance was 212 measured by annotating remainder of data. HMMER based methods were tested with $25 \%$ of 213 random sequences from input datasets, using all appropriate HMM models. Database 214 construction and testing was repeated 10 times and results were averaged.

215 ToxClassifier Meta-classifier calibration and testing 
216 ToxClassifier meta-classifier was constructed from nine annotation model and classifier 217 combinations (BIF_SVM, BIF_GBM, STB_SVM, STB_GBM, TBS_SVM, TBS_SVM, 218 TBEa_SVM, TBEb_SVM and TBEb_GBM). Each of nine classifiers reports 1 if the input 219 sequence is predicted as toxin or 0 if predicted as non-toxic, for final prediction score of 0 to 9. 220 Datasets used for calibration of meta-classifier were chosen from the set of venomous and non221 venomous animals (human Homo sapiens, the house mouse Mus musculus, the Burmese python 222 Python bivittatus, king cobra Ophiophagus hannah, the duck-billed platypus Ornithorhynchus 223 anatinus, the snakelocks sea anemone Anemonia viridis, the starlet sea anemone Nematostella 224 vectensis; and all proteins deposited in the UniProtKB/SwissProt and TrEMBL databases 225 attributed to snakes, spiders, wasps and Conus snails). All sequences were downloaded from 226 UniProtKB database with exception of Python bivittatus which was not available in UniProtKB 227 and was downloaded from NCBI protein database (Wheeler et al. 2003); all data is available at 228 https:/github.com/rgacesa/ToxClassifier/datasets. Datasets were split into training sets 229 consisting of $75 \%$ of data and test sets including remaining $25 \%$ of sequences.

230 ToxClassifier meta-classifier was calibrated by evaluating prediction score versus performance

231 for each animal training set and for summary dataset constructed by combining animal training 232 datasets with exclusion of Conus snail data, which was dropped due to suspected low quality of 233 annotation. Calibrated ToxClassifier, with prediction score 5 or more as cut-off for positive 234 classification, was tested on animal data test sets, and performance measures were compared to 235 OneBLAST, NaiveBLAST models and ClanTox server. Data used for training and testing and all 236 calculated metrics are available at

237 https://github.com/rgacesa/ToxClassifier/datasets/toxclassifier_calibration test.

238 ToxClassifier comparison to other published tools 
239 Performance of ClanTox server (Kaplan et al. 2007) was tested on Positive, Easy, Moderate and

240 Hard datasets and on animal data test sets. ToxinPred server (Gupta et al. 2013) was tested on

241868 Positive dataset sequences of length up to 30 amino acids (ToxinPred sequence length limit)

242 and on negative dataset composed 30 amino acid or shorter protein sequences randomly selected

243 from UniProt database (5,673 non-duplicate, non-ToxProt sequences). ToxinPred was not tested

244 on animal data due to lack of short sequences available in these datasets. SpiderP server (Wong

245 et al. 2013) was not tested as service was not available at the time and PredCSF server (Fan et al.

246 2016) was not tested as it was deemed too specialized and only accepts single sequence as input.

247 User interface

248 The ToxClassifier web service front-end is implemented using HTML 5.1

249 (https://www.w3.org/html/), JavaScript (https://www.javascript.com/), jQuery

250 (https://jquery.com/), $\quad$ CSS $\quad$ (https://www.w3.org/Style/CSS/), $\quad$ Java $\quad 7.0$

251 (https://www.oracle.com/java/index.html) and the Java Server Pages (JSP 2.1) framework

252 (http://www.oracle.com/technetwork/java/javaee/jsp/index.html). Visualisation is performed

253 using $\mathrm{R}$ (https://www.r-project.org/), ggplot2 (http://ggplot2.org/) and R markdown

254 (http://rmarkdown.rstudio.com/) packages. ToxClassifier runs on an Apache Tomcat 8.0 web

255 server (http://tomcat.apache.org/download-80.cgi), under an Ubuntu Linux 12.04 operating

256 system (http://www.ubuntu.com/). The service is hosted by the Section of Bioinformatics,

257 Faculty of Food Technology and Biotechnology, University of Zagreb, Croatia

258 (http://www.pbf.unizg.hr/en/departments/department_of biochemical_engineering/section_for_b

259 ioinformatics).

260 Results 
262 having other physiological functions was assessed by training each classifier using 7 different

263 annotation models. The learning classifiers were a Support Vector Machine (SVM) and Gradient

264 Boosted Machine (GBM) chosen as high-performing predictors, and a Generalised Linear Model

265 (GLM) regarded as a simple classifier, but with which a baseline could be established that would

266 allow comparison of the performance of the SVM and GBM machines. A detailed description of

267 the annotation models is given in Methods section, but briefly the annotation models used the

268 following sequence information from the training set as classifier inputs: either the frequency of

269 amino acids (TBSim) or combinations of two amino-acids (BIF), the presence of absence or

270 'tox-bits' (SToxA), HMM scores for 'tox-bits' (SToxB), a selection of BLAST output co-

271 variants (TBEa) or a variation on TBSim and TBEa (TBEb).

272 The training set was constructed by merging $75 \%$ of arbitrarily selected sequences from

273 each of the following datasets: 1/ A 'Positive' dataset that contained all 8,093 protein sequences

274 deposited in the UniProtKB/SwissProt-ToxProt database of animal venom toxins (Jungo et al.

275 2012). 2/ An 'Easy' dataset composed of 47,144 random, non-duplicate sequences from

276 UniProtKB/SwissProt database (Bateman et al. 2015). 3/ A 'Moderate' dataset comprised of

2778,034 unique sequences from the manually curated UniProtKB/SwissProt database considered to

278 be non-toxic but with homology to toxin proteins in UniProtKB/SwissProt-ToxProt. 4/ A 'Hard'

279 dataset that included 7,403 non-duplicate sequences extracted from the computer annotated

280 TrEMBL (Bairoch \& Apweiler 2000) database, also considered to be non-toxic but with

281 homology to animal venom toxins in UniProtKB/SwissProt-ToxProt.

282 All training was performed using 10 internal bootstrap cross-validations on the training

283 set, and learning curves showing the accuracy of predictions versus the number of sequences in 
284 the training sets were constructed, thereby allowing a comparative evaluation of training

285 efficiency (Fig. S1). The trained classifiers were then tested for prediction accuracy using the

286 remaining $25 \%$ of sequences not included in the training set. Performance values were calculated

287 to give an overall comparative classification of protein sequences as toxins or non-toxins (Table

288 1). By comparing learning curves (Fig. S1) and accuracy of predictions (Table 1), 9 of the

289 annotation model and classifier combinations were chosen to construct the 'ToxClassifier'

290 ensemble. The trained classifiers were: BIF_SVM, BIF_GBM, STB_SVM, STB_GBM,

291 TBS_SVM, TBS_SVM, TBEa_SVM, TBEb_SVM and TBEb_GBM. These classifiers all gave

292 excellent accuracy scores to predict toxins from the Positive dataset (range 0.82 to 0.96) and

293 non-toxin proteins from the Easy, Moderate and Hard datasets (range 0.92 to 1.00). No GLM

294 classifiers were included in the ensemble because prediction accuracies were considerably lower

295 when compared with SVM and GBM machines. Classifiers using NTB and OF annotation

296 models were also abandoned in favour of better performing STB and BIF models. Furthermore,

297 the TBEa_GBM model consistently underperformed compared to the TBE_SVM model and was

298 excluded, giving an odd number of classifiers in the ensemble, thereby avoiding a 'tied vote'

299 scenario when the outputs were interpreted collectively.

300 The prediction accuracy of the trained machine learning classifiers was next compared to

301 more conventional annotation methods based on sequence alignment, to determine if machine

302 learning predictions were superior or inferior to well established and accepted bioinformatics

303 tools. A detailed description of the annotation models based on these bioinformatics tools is

304 given in Methods. Briefly, simple predictions were made by taking the best-hit from BLAST

305 comparisons between a query sequence and the UniProtKB/SwissProt-ToxProt database

306 (naiveBLAST method), or the best-hit following a HMMER hmmsearch comparison between a 
307 query sequence and either existing HMM models for toxin protein families in the Pfam database

308 (hmmerVenom model), or our own 'tox-bits' HMM models (hmmerToxbits classifier). More

309 sophisticated annotation models also used BLAST or HMMER searches, but the best-hit was

310 extracted following simultaneous comparisons between the query sequence and multiple

311 datasets. These sophisticated annotation models are also described in Methods, but briefly these

312 models were constructed from sequence information extracted from either

313 UniProtKB/SwissProt-ToxProt sequences supplemented with additional toxin-like sequences

314 from the UniProtKB/SwissProt and TrEMBL databases, or UniProtKB/SwissProt-ToxProt

315 sequences supplemented with non-toxin sequences from the 'Moderate' and 'Hard' datasets used

316 to train the machine classifiers. Training and test sets were analogous in design and execution to

317 the machine classifier learning, with $75 \%$ of sequence information used to construct the BLAST

318 and HMMER databases and the remaining $25 \%$ of data used to evaluate performance. Prediction

319 accuracy measures for each query sequence using each of the bioinformatics models were

320 repeated 10 times to give a final balanced accuracy value. Accuracy measure calculations are

321 described in Methods. A range of sequence-alignment scoring was also tested to select the lowest

322 BLAST and HMMER cut-off scores that gave the most precise toxin annotation. This value was

323 1.0e-20 for both BLAST and HMMER searches (Fig. S2).

324 Machine learning classifiers were also evaluated against currently available published 325 tools for toxin prediction and annotation; Animal toxin prediction server ClanTox (Kaplan et al. 326 2007) was benchmarked using Positive, East, Moderate and Hard datasets and summary of these

327 datasets. As ToxinPred (Gupta et al. 2013) tools predict only small peptide toxins, it was tested

328 using a subset of Positive dataset with sequences no longer than 30 amino acids (868 sequences)

329 and separate negative dataset composed of 5,673 random short proteins from UniProtKB 
330 database. SpiderP (Wong et al. 2013) was not benchmarked as the server no longer seems

331 publically available. Finally, PredCSF (Fan et al. 2016) is a conotoxin-specific tool and was

332 deemed not comparable to general annotation tools; it also only allows single sequence input,

333 making it unsuitable for large scale testing. Final performance measures compared between the

334 different tools are listed in Table 2.

335 Testing of sequence-alignment based annotation models (Table 2) demonstrated that the 336 simplistic methods (naiveBLAST, hmmerToxBits and hmmerVenom) gave high prediction

337 accuracies for sequences in the Easy dataset (ranging from 0.95 for hmmerVenom to 0.99 for

338 naiveBLAST), but underperformed in annotation of the physiological toxin-like sequences in the

339 Moderate and Hard datasets (accuracies ranging from 0.74 to 0.83 for Moderate and 0.07 to 0.23

340 for Hard dataset (the poor performance here, also evinced by the low F1 and MCC scores). More

341 sophisticated BLAST-based methods (oneBLAST and triBLAST) gave very high prediction

342 accuracy scores $(0.93-0.999)$ for sequences in the Easy and Moderate datasets, but somewhat

343 lower performance on sequences in the Positive and Hard datasets $(0.86-0.90)$. Pfam-based

344 twinHMMER gave the highest accuracy prediction for non-toxin sequences, but underperformed

345 compared to the other annotation models against sequences in the positive toxin dataset

346 (accuracy 0.56). The 'tox-bits' based variant accurately predicted sequences in the Easy and

347 Moderate datasets (accuracy $0.85-0.999$ ), but suffered from a high false positive rate when

348 sequences in the Hard dataset were analysed (accuracy 0.44). When compared to machine

349 learning-based methods, even the most accurate of the sequence alignment-based models

350 (oneBLAST and triBLAST) were surpassed by the majority of the machine learning based

351 classifiers, especially by TBEa and TBEb models (SVM and GBM variants), which gave the

352 highest accuracy of prediction for sequences in all test datasets. All prediction methods showed 
353 higher performance for negative prediction (predicting non-toxin as non-toxin) compared to

354 positive prediction (correctly predicting toxin as toxic), with Specificity (Spec) and Negative

355 Prediction Value (NPV) significantly higher than Sensitivity (Sens) and Positive Prediction

356 Value (PPV).

Each of the 9 machine learning classifiers used in the 'ToxClassifier' ensemble gives a

358 simple bit ( 1 or 0$)$ value as output to predict whether the likely biological activity of the input

359 sequence is as a toxin (1), or has a non-toxic (0) physiological role and scores are summed into

360 final prediction score ranging from 0 to 9 . Evaluation of this final prediction score was

361 performed on test sets obtained from randomly sampling $75 \%$ of sequences in the published

362 annotated genomes from a selection of venomous animals (king cobra Ophiophagus hannah, the

363 duck-billed platypus Ornithorhynchus anatinus, the snakelocks sea anemone Anemonia viridis,

364 the starlet sea anemone Nematostella vectensis; and all proteins deposited in the

365 UniProtKB/SwissProt and TrEMBL databases attributed to snakes, spiders, wasps and Conus

366 snails) and other animals considered to be non-venomous (human Homo sapiens, the house

367 mouse Mus musculus and the Burmese python Python bivittatus). Calibration was performed by

368 assessing the performance measures of the Toxclassifier ensemble relative to prediction score;

369 calibration curves for summary of all animal genome data are presented in Figure 1. When the

370 average correct annotation of all input sequences for all genomes was calculated, a combined

371 score from 5 out of the 9 classifiers giving correct classification provided a good balance

372 between the detection of toxins and the filtering of non-toxins. Hence, a calibration for the

373 ToxClassifier ensemble was possible where an input sequence giving a combined score of $>6$

374 would be considered a likely toxin, a combined score of $<3$ would be regarded as non-toxic,

375 while an input sequence presenting with a score 4 or 5 would suggest a potential toxin, but would 
376 require manual evaluation using additional tools, for example, InterProScan (Zdobnov \& 377 Apweiler 2001).

378 Performance of calibrated 'ToxClassifier' meta-classifier was evaluated on a test set 379 comprising $25 \%$ of the animal genome data not used for calibration; these results were compared 380 to naiveBLAST and OneBLAST conventional methods and to ClanTox server for animal toxin 381 prediction (Kaplan et al. 2007). Performance measurements are reported in Table 3 and 382 comparison of F1-scores and MCC values across all datasets is presented in Figures 2, S3/A and

383 S3/B. Finally, the 'ToxClassifier' was assessed in a blinded experiment that used as input a set of 384 protein sequences derived from the venom gland transcriptome of the Amazonian rain forest pit 385 viper Bothrops atrox (Supplementary Data 1). The sequences had been annotated using standard 386 methods and manually inspected, with the biological activities of some also being authenticated 387 experimentally. The results of the 'ToxClassifier' predictions matched with the expert annotation 388 (Table 4).

389 Discussion

The continued decline in proteomics sequencing costs over recent years has led to an

391 explosion in venomics data characterising the toxic peptide and protein components in many

392 venomous animals (Kaas \& Craik 2015). However, there is currently no widely accepted and 393 standard method for functional annotation of toxins from these data sources, leading to 394 inconsistent estimates for the number of toxins in the venom of the same animal. For example, 395 the venom of the duck-billed platypus Ornithorhynchus anatinus has only 6 toxins listed 396 following manual annotation in the latest release of the UniProtKB/SwissProt-ToxProt database 397 (11th May 2016), yet 107 putative toxins were identified by a simple pair-wise BLASTp search 
398 using venom gland transcriptome sequences as input to search the UniProtKB/SwissProt ToxProt

399 database (Jungo et al. 2012). In addition to separate homology searching methods to interpret the

400 same data, many venomics projects now also include different manual filtering steps as part of

401 the annotation process (Rachamim et al. 2014; Gacesa et al. 2015), exacerbating the problem of 402 results verification.

403 In this study, a selection of machine learning-based classifiers implementing a range of

404 BLAST and HMMER-based annotation models were trained on datasets of known toxins,

405 protein sequences assumed to be non-toxic but with homology to known toxins, and predicted

406 proteins encoded in the genome, transcriptome or proteome of a range of venomous and non-

407 venomous animals. A comparison between the results presented in Tables 1-3 demonstrated that

408 the majority of the machine learning methods consistently out-performed standard bioinformatics

409 approaches of functional annotation. Interestingly, all tested methods demonstrated higher

410 performance for negative prediction (classification of non-toxic sequences) compared to positive

411 classification (prediction of toxic sequences as toxins). These results demonstrate that

412 differentiating between physiological toxin-like proteins and actual toxins is more difficult then

413 prediction of random proteins as non-toxic, which is to be expected considering the similarity

414 and common origin of many toxins and toxin-like sequences (Fry 2005; Chang \& Duda 2012;

415 Hargreaves et al. 2014). As such, it is important to consider balanced performance measurements

416 when assessing toxin classifiers, with scores such as F1-score and MCC value (Matthews 1975;

417 Powers 2011) providing more appropriate measurements of performance than simple accuracy.

418 Another issue of toxin classification lies in unbalanced datasets, because most venomous animal

419 genomes encode less than 100 toxins and 20,000 to 30,000 physiological non-toxic proteins; as a

420 result, even a high performing method can generate a high number of false positive predictions. 
421 For example, $99.5 \%$ correct prediction of non-toxins results in $\sim 100$ false positive toxins for an

422 average animal proteome, which is in fact more than the actual number of true toxins. In order to

423 minimize both of these problems, the ToxClassifier training scheme was conservative, using only

424 well-annotated toxins from UniProtKB/ToxProt database as positives, and while this might lead

425 to a somewhat lower positive prediction rate (due to missing likely toxins which are not

426 annotated as such), it does serve to minimise the false positive rate.

427 Notably, predictions were less accurate on some genome datasets, especially Conus snail

428 proteins, with low performance metrics observed for all tested annotation methods. This

429 discrepancy was likely caused by the assumption that sequences deposited in the

430 UniProtKB/SwissProt-ToxProt sequence are bona fide toxins, while sequences in the

431 UniProtKB/SwissProt and TrEMBL databases without 'toxin' or 'venom' keywords are not

432 toxins. Given that toxin activity is attributed to most sequences without biological validation, it is

433 likely that the training datasets almost certainly excluded a number of toxin sequences and

434 included some yet unknown toxins as non-toxic. Another limitation of the 'ToxClassifier' lies in

435 the inherent bias of the training sets; an underrepresentation in sequences from certain animal

436 lineages, particularly the basal Metazoa, e.g. Cnidaria, could lead to incorrect assignment and

437 suspicious quality of existing annotation of conotoxins is a reason to treat prediction on this

438 protein class with caution. To elevate these problems, 'ToxClassifier' has been designed to also

439 report sequences suspected to have closest homology to underrepresented taxa as 'suspicious

440 toxin' and recommends manual annotation with other tools, such as InterProScan (Zdobnov \&

441 Apweiler 2001).

442 Use of machine learning for toxin prediction has been attempted before and a range of

443 such tools exists; however, most of the available tools are heavily specialised for toxins of 
444 specific animal origins. For example, SpiderP (Wong et al. 2013)

445 (http://www.arachnoserver.org/spiderP.html) is a predictor for spider toxins while ToxinPred

446 (Gupta et al. 2013) (http://crdd.osdd.net/raghava/toxinpred/) predicts only small peptide toxins;

447 while ClanTox (Kaplan et al. 2007) (http://www.clantox.cs.huji.ac.il/tech.php) was trained only

448 on an ion-channel toxin dataset and PredCSF (Fan et al. 2016)

449 (http://www.csbio.sjtu.edu.cn/bioinf/PredCSF/) is conotoxin specific. In addition, the reported

450 training set sizes are low (for example ClanTox was trained on $\sim 600$ ion channel toxins; the

451 ToxinPred toxin positive training set is 1805 sequences, while as of 11th May 2016, the

452 UniProtKB/SwissProt-ToxProt database contained $\sim 6500$ sequences). None of the currently

453 available machine learning methods also gives a comparison with other currently used accepted

454 bioinformatics annotation methods. When compared to ToxClassifier and conventional

455 annotation tools (Tables 2 and 3), ClanTox and ToxinPred tools were found to perform similar to

456 BLAST based methods, while ToxClassifier demonstrated higher performance across all metrics,

457 which is likely a result of comparatively larger training sets and combination of different internal 458 classifiers.

459 In addition to high performance, the user interface of the 'ToxClassifier' web service 460 reports the best-scoring hit annotation either to UniProtKB/SwissProt-ToxProt (allowing 461 placement of the toxin into the most appropriate toxin protein family), or to the best hit in 462 UniProtKB/SwissProt (giving the closest homology to a non-toxin protein). In summary, this 463 study has established baseline prediction accuracies for a selection of toxin annotation methods 464 and integrates these methods into an easy-to-use, high-precision, machine learning-based 465 classification system named 'ToxClassifier'.This tool offers a reliable and reproducible 
466 framework for toxin annotation to enable standardised toxin prediction in venomics projects and

467 to allow for semi-automatic annotation or re-annotation of existing datasets.

\section{References}

469 Altschul SF., Madden TL., Schäffer AA., Zhang J., Zhang Z., Miller W., Lipman DJ. 1997.

470 Gapped BLAST and PSI-BLAST: a new generation of protein database search programs. Nucleic Acids Research 25:3389-3402. DOI: 10.1093/nar/25.17.3389.

Bairoch A., Apweiler R. 2000. The SWISS-PROT protein sequence database and its supplement TrEMBL in 2000. Nucleic Acids Research 28:45-48. DOI: 10.1093/nar/28.1.45.

Bateman A., Martin MJ., O’Donovan C., Magrane M., Apweiler R., Alpi E., Antunes R., 
489

490

491

492

493

494

495

496

497

498

499

500

501

502

503

504

505

506

507

508

509

510

511

Verbregue L., Veuthey AL., Wu CH., Arighi CN., Arminski L., Chen C., Chen Y., Garavelli JS., Huang H., Laiho K., McGarvey P., Natale DA., Suzek BE., Vinayaka CR., Wang Q., Wang Y., Yeh LS., Yerramalla MS., Zhang J. 2015. UniProt: A hub for protein information. Nucleic Acids Research 43:D204-D212. DOI: 10.1093/nar/gku989.

Bromberg Y., Yachdav G., Ofran Y., Schneider R., Rost B. 2009. New in protein structure and function annotation: hotspots, single nucleotide polymorphisms and the "Deep Web". Current Opinion in Drug Discovery \& Development 12:408-419.

Camacho C., Coulouris G., Avagyan V., Ma N., Papadopoulos J., Bealer K., Madden TL. 2009. BLAST+: architecture and applications. BMC Bioinformatics 10:1-9. DOI: 10.1186/14712105-10-421.

Chang D., Duda TF. 2012. Extensive and continuous duplication facilitates rapid evolution and diversification of gene families. Molecular Biology and Evolution 29:2019-2029. DOI: 10.1093/molbev/mss068.

Eddy SR. 2011. Accelerated profile HMM searches. PLoS Computational Biology 7:e1002195. DOI: 10.1371/journal.pcbi.1002195.

Fan YX., Song J., Kong X., Shen H Bin. 2016. PredCSF: An integrated feature-based approach for predicting conotoxin superfamily. Protein \& Peptide Letters 18:261-267. DOI: $10.2174 / 092986611794578341$.

Fry B. 2005. From genome to "venome": molecular origin and evolution of the snake venom proteome inferred from phylogenetic analysis of toxin sequences and related body proteins. Genome Research 15:403-420. DOI: 10.1101/gr.3228405.

Gacesa R., Chung R., Dunn SR., Weston AJ., Jaimes-Becerra A., Marques AC., Morandini AC., Hranueli D., Starcevic A., Ward M., Long PF. 2015. Gene duplications are extensive and 
512 contribute significantly to the toxic proteome of nematocysts isolated from Acropora

513 digitifera (Cnidaria: Anthozoa: Scleractinia). BMC Genomics 16:774. DOI:

$514 \quad 10.1186 / \mathrm{s} 12864-015-1976-4$.

515 Gupta S., Kapoor P., Chaudhary K., Gautam A., Kumar R., Raghava GPS. 2013. In silico

516 approach for predicting toxicity of peptides and proteins. PLOS ONE 8, e73957 DOI:

$517 \quad$ 10.1371/journal.pone.0073957.

518 Hargreaves AD., Swain MT., Hegarty MJ., Logan DW., Mulley JF. 2014. Restriction and

519 recruitment - gene duplication and the origin and evolution of snake venom toxins. Genome

520 Biology and Evolution 6: 2088-2095. DOI: 10.1093/gbe/evu166.

521 Jungo F., Bougueleret L., Xenarios I., Poux S. 2012. The UniProtKB/Swiss-Prot Tox-Prot

522 program: A central hub of integrated venom protein data. Toxicon 60:551-557. DOI:

$523 \quad 10.1016 /$ j.toxicon.2012.03.010.

524 Junqueira-de-Azevedo ILM., Bastos CMV., Ho PL., Luna MS., Yamanouye N., Casewell NR.

525 2015. Venom-related transcripts from Bothrops jararaca tissues provide novel molecular

526 insights into the production and evolution of snake venom. Molecular Biology and

527 Evolution 32:754-66. DOI: $10.1093 / \mathrm{molbev} / \mathrm{msu} 337$.

528 Kaas Q., Craik DJ. 2015. Bioinformatics-aided venomics. Toxins 7:2159-2187. DOI:

$529 \quad 10.3390 /$ toxins 7062159.

530 Kaplan N., Morpurgo N., Linial M. 2007. Novel families of toxin-like peptides in insects and

531 mammals: A computational approach. Journal of Molecular Biology 369:553-566. DOI:

$532 \quad 10.1016 /$ j.jmb.2007.02.106.

533 Krogh A., Brown M., Mian IS., Sjölander K., Haussler D. 1994. Hidden markov models in 534 computational biology, applications to protein modeling. Journal of Molecular Biology 
235:1501-1531. DOI: 10.1006/jmbi.1994.1104.

536 Li R., Yu H., Xing R., Liu S., Qing Y., Li K., Li B., Meng X., Cui J., Li P. 2012. Application of

537 nanoLC-MS/MS to the shotgun proteomic analysis of the nematocyst proteins from jellyfish

538 Stomolophus meleagris. Journal of Chromatography B: Analytical Technologies in the

539 Biomedical and Life Sciences 899:86-95. DOI: 10.1016/j.jchromb.2012.05.006.

540 Li R., Yu H., Xue W., Yue Y., Liu S., Xing R., Li P. 2014. Jellyfish venomics and venom gland

541 transcriptomics analysis of Stomolophus meleagris to reveal the toxins associated with

542 sting. Journal of Proteomics 106:17-29. DOI: 10.1016/j.jprot.2014.04.011.

543 Liu G., Zhou Y., Liu D., Wang Q., Ruan Z., He Q., Zhang L. 2015. Global transcriptome

$544 \quad$ analysis of the tentacle of the Jellyfish Cyanea capillata using deep sequencing and

545 expressed sequence tags: Insight into the toxin-and degenerative disease-related transcripts.

$546 \quad$ PLoS ONE 10:1-22. DOI: 10.1371/journal.pone.0142680.

547 Matthews BW. 1975. Comparison of the predicted and observed secondary structure of T4 phage

548 lysozyme. BBA - Protein Structure 405:442-451. DOI: 10.1016/0005-2795(75)90109-9.

549 Neumann RS., Kumar S., Shalchian-Tabrizi K. 2014. BLAST output visualization in the new

$550 \quad$ sequencing era. Briefings in Bioinformatics 15:484-503. DOI: 10.1093/bib/bbt009.

551 Powers D. 2011. Evaluation: From precision, recall and f-measure to roc., informedness,

552 markedness \& correlation. Journal of Machine Learning Technologies 2:37 - 63.

553 Rachamim T., Morgenstern D., Aharonovich D., Brekhman V., Lotan T., Sher D. 2014. The

554 dynamically evolving nematocyst content of an anthozoan, a scyphozoan, and a hydrozoan.

555 Molecular Biology and Evolution 32:740-753. DOI: 10.1093/molbev/msu335.

556 Reyes-Velasco J., Card DC., Andrew AL., Shaney KJ., Adams RH., Schield DR., Casewell NR.,

557 Mackessy SP., Castoe TA. 2015. Expression of venom gene homologs in diverse python 
tissues suggests a new model for the evolution of snake venom. Molecular Biology and Evolution 32:173-183. DOI: 10.1093/molbev/msu294.

560 Schwartz EF., Diego-Garcia E., Rodríguez de la Vega RC., Possani LD., Whittington CM., 561 Papenfuss AT., Locke DP., Mardis ER., Wilson RK., Abubucker S., Mitreva M., Wong 562 ESW., Hsu AL., Kuchel PW., Belov K., Warren WC., Sher D., Zlotkin E., Knebel A., Bsor 563 T., Nesher N., Tal T., Morgenstern D., Cohen E., Fishman Y., Zlotkin E., Schwartz EF., 564 Diego-Garcia E., Rodríguez de la Vega RC., Possani LD., Liu G., Zhou Y., Liu D., Wang 565 Q., Ruan Z., He Q., Zhang L. 2015. Transcriptome analysis of the venom gland of the 566 Mexican scorpion Hadrurus gertschi (Arachnida: Scorpiones). Toxicon 11:865-879. DOI: 10.1371/journal.pone.0142680.

Sher D., Zlotkin E. 2009. A hydra with many heads: Protein and polypeptide toxins from hydra and their biological roles. Toxicon 54:1148-1161. DOI: 10.1016/j.toxicon.2009.02.036.

Starcevic A., Moura-Da-Silva AM., Cullum J., Hranueli D., Long PF. 2015. Combinations of long peptide sequence blocks can be used to describe toxin diversification in venomous animals. Toxicon 95:84-92. DOI: 10.1016/j.toxicon.2015.01.005.

Starcevic A., Long PF. 2013. Diversification of animal venom peptides-were jellyfish amongst the first combinatorial chemists? ChemBioChem 14:1407-1409. DOI:

Vonk FJ., Casewell NR., Henkel C V., Heimberg AM., Jansen HJ., McCleary RJR., Kerkkamp HME., Vos R a., Guerreiro I., Calvete JJ., Wüster W., Woods AE., Logan JM., Harrison R a., Castoe T a., de Koning a PJ., Pollock DD., Yandell M., Calderon D., Renjifo C., Currier RB., Salgado D., Pla D., Sanz L., Hyder AS., Ribeiro JMC., Arntzen JW., van den Thillart GEEJM., Boetzer M., Pirovano W., Dirks RP., Spaink HP., Duboule D., McGlinn E., Kini 
581

582

583

RM., Richardson MK. 2013. The king cobra genome reveals dynamic gene evolution and adaptation in the snake venom system. Proceedings of the National Academy of Sciences of the United States of America 110:20651-20656. DOI: 10.1073/pnas.1314702110.

Weston AJ., Dunlap WC., Shick JM., Klueter A., Iglic K., Vukelic A., Starcevic A., Ward M., Wells ML., Trick CG., Long PF. 2012. A profile of an endosymbiont-enriched fraction of the coral Stylophora pistillata reveals proteins relevant to microbial-host Interactions. Molecular \& Cellular Proteomics 11:M111.015487-M111.015487. DOI: 10.1074/mcp.M111.015487.

Weston AJ., Chung R., Dunlap WC., Morandini AC., Marques AC., Moura-da-Silva AM., Ward M., Padilla G., da Silva LF., Andreakis N., Long PF. 2013. Proteomic characterisation of toxins isolated from nematocysts of the South Atlantic jellyfish Olindias sambaquiensis. Toxicon 71:11-17. DOI: 10.1016/j.toxicon.2013.05.002.

Wheeler DL., Church DM., Federhen S., Lash AE., Madden TL., Pontius JU., Schuler GD., Schrimi LM., Sequerira E., Tatusova TA., Wagner L. 2003. Database resources of the National Center for Biotechnology. Nucleic Acids Research 31:28-33. DOI: 10.1093/nar/gkg033.

Whittington CM., Papenfuss AT., Locke DP., Mardis ER., Wilson RK., Abubucker S., Mitreva M., Wong ESW., Hsu AL., Kuchel PW., Belov K., Warren WC. 2010. Novel venom gene discovery in the platypus. Genome Biology 11:R95. DOI: 10.1186/gb-2010-11-9-r95.

Wong ESW., Hardy MC., Wood D., Bailey T., King GF. 2013. SVM-based prediction of propeptide cleavage sites in spider toxins identifies toxin innovation in an Australian tarantula. PloS One 8:e66279. DOI: 10.1371/journal.pone.0066279.

Zdobnov EM., Apweiler R. 2001. InterProScan-an integration platform for the signature- 
604

605

606

607

608

609

610

611

612

613

614

615

616

617

618

619

620

621

622

623

624

625

626 recognition methods in InterPro. Bioinformatics (Oxford, England) 17:847-848. DOI: 10.1093/bioinformatics/17.9.847.

\section{Acknowledgments}

The authors are grateful to Prof. Dr. Ana M. Moura-da-Silva who provided the unpublished anonymised snake venom transcriptome sequences. We also thank Prof. J. Malcolm Shick and Prof Dr John Cullum for critically reviewing the manuscript.

\section{Authors' Contributions}

PFL conceived the study. RG carried out the bioinformatics analysis. All authors interpreted the data, wrote the manuscript and have approved the final version of the manuscript.

\section{Competing financial interests}

The authors declare that they have no competing interests.

Figure 1 legend: Figure displays calibration curves used to select final prediction scores for ToxClassifier ensemble. Each of performance measures, described in detail in Methods, is shown for ToxClassifier prediction with toxin prediction cut-off values 1 to 9 . Dotted green line shows trends of prediction measurement with increase in ToxClassifier cut-off value and final cut-off value implemented in calibrated ToxClassifier is highlighted with blue line.

Figure 2 legend: Figure shows comparison between selected toxin prediction tools for all animal test datasets. Calibrated ToxClassifier, with positive prediction cutoff 5, is shown by green bar, 
627 ClanTox prediction server is displayed in orange, oneBLAST prediction performance in red and

628 naiveBLAST in blue. F1-value and Matthews' correlation coefficient are displayed for each of

629 test sets and for summary of all data with exclusion of conus snail proteins.

630

631 Table 1 legend: Table displays prediction accuracy on positive and negative datasets, as well as

632 range of measurements calculated for all test data, and described in detail in Methods.

633 Annotation models used as classifier inputs either: the frequency of amino acids (TBSim) or

634 combinations of two amino-acids (BIF); the presence of absence or 'Tox-Bits' (SToxA); HMM

635 scores for 'ToxBits' (SToxB); a selection of BLAST output co-variants (TBEa); a variation on

636 TBSim and TBEa (TBEb). Classifier Learning Machines used were: Gradient Boosted (GBM),

637 Support Vector (SVM) and Generalised Linear Model (GLM). The datasets were a 'Positive'

638 control containing only validated animal toxins, an 'Easy' dataset composed of non-toxin

639 sequences, a 'Moderate' dataset comprising curated non-toxin sequences but with homology to

640 'Positive' sequences, and a 'Hard' dataset that included all sequences from the 'Moderate'

641 dataset, together with un-curated sequences also with homology to 'Positive' sequences.

642

643 Table 2 legend: Table compares performance for selected annotation models and published

644 toxin prediction tools. Prediction accuracy is listed for positive and negative datasets and

645 measurements are also shown for summary of all test data. ToxinPred tool, marked with star and

646 displayed in italic, was tested with short protein sequences only. Annotation models were

647 constructed from sequence information extracted from either: BLAST (naiveBLAST), 'tox-bits'

648 HMM (hmmerToxBits) or Pfam HMM (hmmerVenom) comparisons with the

649 UniProtKB/SwissProt-ToxProt database; or BLAST (oneBLAST), 'tox-bits' HMM 
650 (twinHmmerPfam) or Pfam HMM (twinHmmerPfam) comparisons with the

651 UniProtKB/SwissProt-ToxProt sequences supplemented with additional toxin-like sequences

652 from the UniProtKB/SwissProt and TrEMBL databases; or BLAST (triBLAST) comparisons

653 with the UniProtKB/SwissProt-ToxProt sequences supplemented with non-toxin sequences from

654 the 'Moderate' and 'Hard' datasets used to train the machine classifiers. The datasets were a 655 'Positive' control containing only validated animal toxins, an 'Easy' dataset composed of non656 toxin sequences, a 'Moderate' dataset comprising curated non-toxin sequences but with 657 homology to 'Positive' sequences, and a 'Hard' dataset that included all sequences from the 658 'Moderate' dataset, together with un-curated sequences also with homology to 'Positive' 659 sequences.

660

661 Table 3 legend: Table lists comparison of classification performance for calibrated 662 ToxClassifier to BLAST based annotation models and ClanTox toxin prediction server. All tests 663 were conducted test dataset not used in calibration of ToxClassifier.

664

665 Table 4 legend: Table compares results of expert manual annotation to ToxClassifier annotation 666 for set of novel proteins from venom gland transcriptome of Bothrops atrox snake. 
Figure 1

Calibration curves used to select final prediction scores for ToxClassifier ensemble

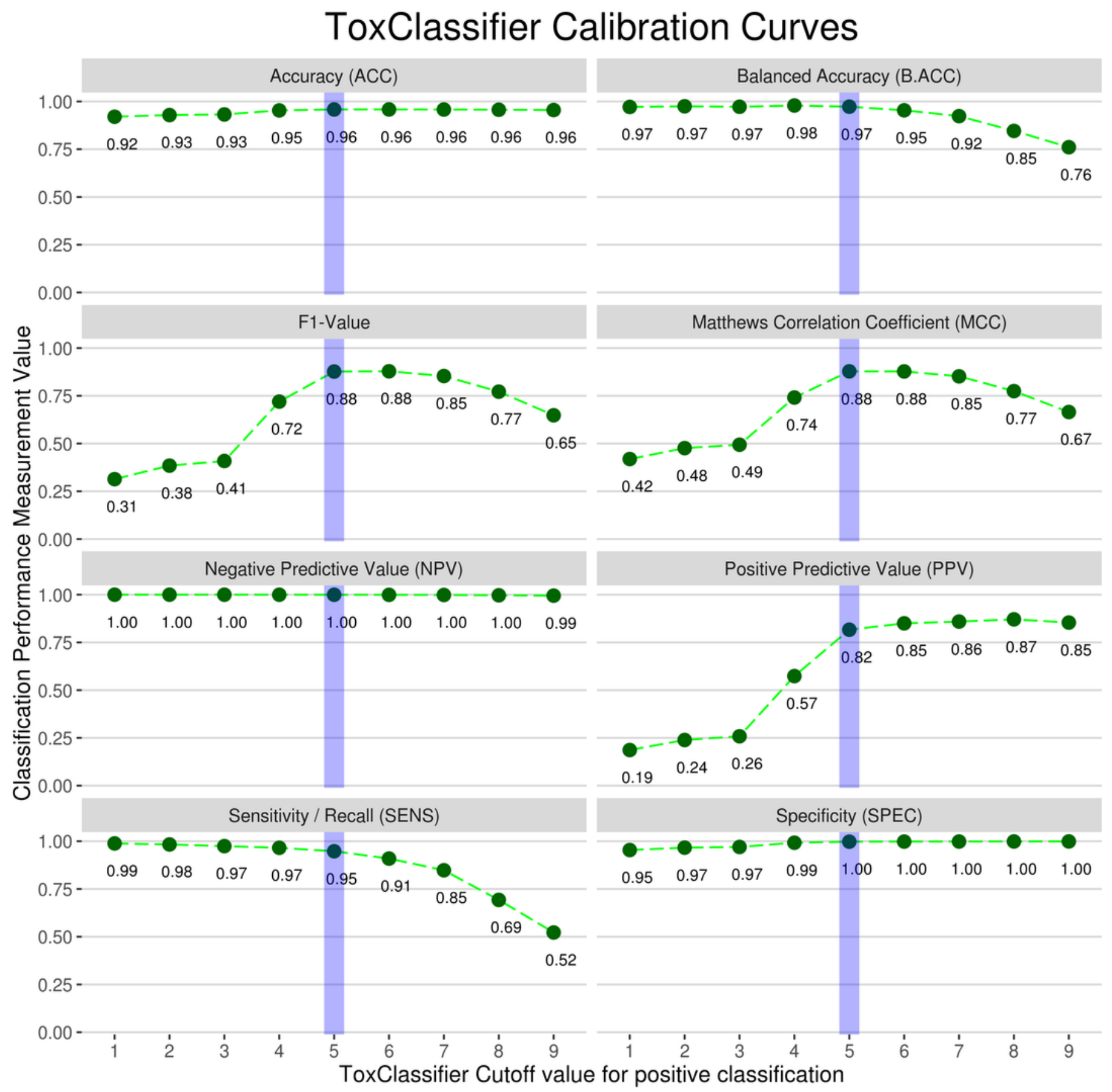


Figure 2

Comparison between selected toxin prediction tools for all animal test datasets.

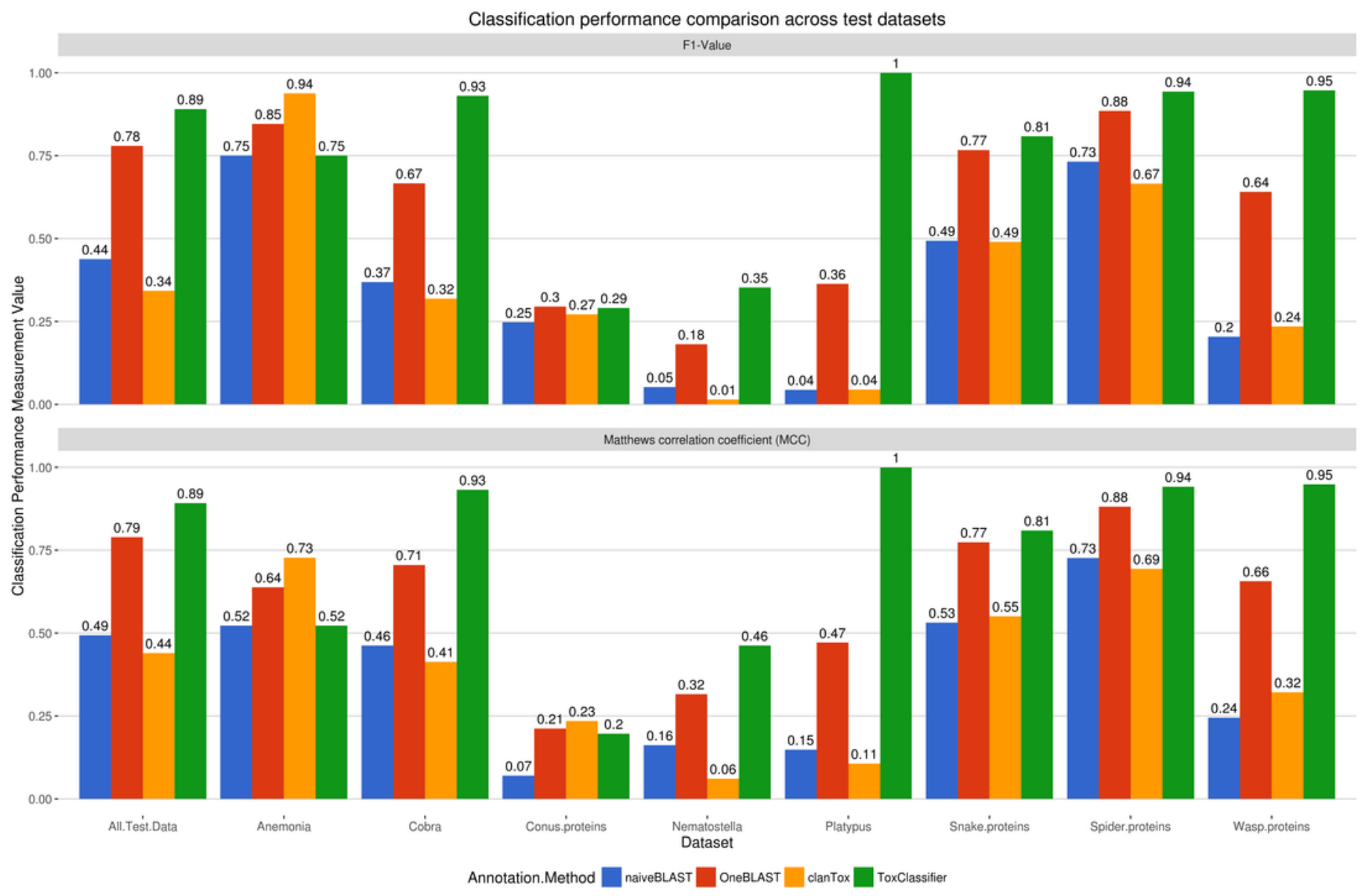




\section{Table $\mathbf{1}$ (on next page)}

Prediction accuracy on positive and negative datasets, as well as range of measurements calculated for all test data 
Table 1: Comparative accuracy values for the classification of protein sequences as toxins or non-toxins using 6 different annotation models trained on 3 separate classifier learning machines.

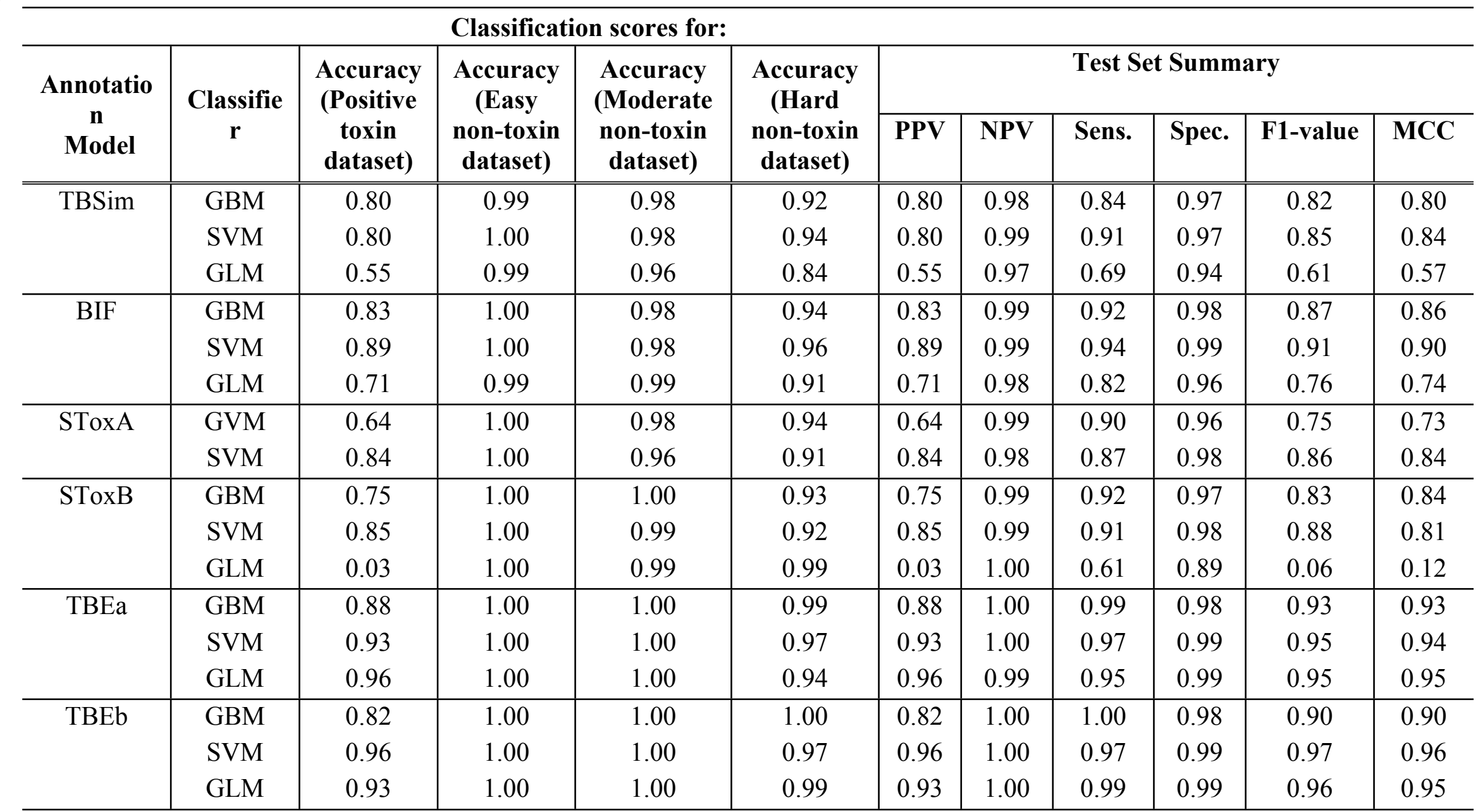

5 


\section{Table 2 (on next page)}

Performance for selected annotation models and published toxin prediction tools. 
Table 2: Comparative balanced accuracy values for classification of protein sequences as toxins or non-toxins using different annotation methodologies based on BLAST or HMMER alignments.

\begin{tabular}{|c|c|c|c|c|c|c|c|c|c|c|c|}
\hline \multirow{3}{*}{$\begin{array}{l}\text { Annotation } \\
\text { Model }\end{array}$} & \multirow[b]{3}{*}{ Tool } & \multirow{3}{*}{$\begin{array}{c}\text { Accuracy } \\
\text { (Positive } \\
\text { toxin } \\
\text { dataset) }\end{array}$} & \multicolumn{9}{|c|}{ Classification scores for: } \\
\hline & & & Accuracy & Accuracy & Accuracy & \multicolumn{6}{|c|}{ Test Set Summary statistics } \\
\hline & & & $\begin{array}{c}\text { non- } \\
\text { toxin } \\
\text { dataset) }\end{array}$ & $\begin{array}{c}\text { e non- } \\
\text { toxin } \\
\text { dataset) }\end{array}$ & $\begin{array}{c}\text { non- } \\
\text { toxin } \\
\text { dataset) }\end{array}$ & PPV & $\begin{array}{c}\text { NP } \\
\text { V }\end{array}$ & Sens. & Spec. & $\begin{array}{c}\text { F1- } \\
\text { value }\end{array}$ & $\mathrm{MCC}$ \\
\hline naiveBLAST & BLAST & 0.90 & 0.99 & 0.83 & 0.07 & 0.90 & 0.86 & 0.46 & 0.99 & 0.60 & 0.58 \\
\hline oneBLAST & BLAST & 0.86 & 1.00 & 0.98 & 0.90 & 0.86 & 0.99 & 0.89 & 0.98 & 0.87 & 0.86 \\
\hline triBLAST & BLAST & 0.87 & 0.99 & 0.93 & 0.87 & 0.87 & 0.97 & 0.78 & 0.98 & 0.82 & 0.80 \\
\hline hmmerToxBits & $\begin{array}{c}\text { HMME } \\
\text { R }\end{array}$ & 0.91 & 0.99 & 0.80 & 0.19 & 0.91 & 0.87 & 0.48 & 0.99 & 0.63 & 0.60 \\
\hline hmmerVenom & $\begin{array}{c}\text { HMME } \\
\text { R }\end{array}$ & 0.65 & 0.95 & 0.74 & 0.23 & 0.65 & 0.84 & 0.34 & 0.95 & 0.45 & 0.38 \\
\hline twinHmmerPfam & $\begin{array}{c}\text { HMME } \\
\text { R }\end{array}$ & 0.56 & 0.99 & 0.98 & 0.91 & 0.56 & 0.98 & 0.78 & 0.95 & 0.65 & 0.62 \\
\hline $\begin{array}{l}\text { twinHmmerToxBi } \\
\text { ts }\end{array}$ & $\begin{array}{c}\text { HMME } \\
\text { R }\end{array}$ & 0.85 & 1.00 & 0.93 & 0.44 & 0.85 & 0.92 & 0.59 & 0.98 & 0.70 & 0.67 \\
\hline ClanTox server & $\mathrm{ML}$ & 0.66 & 0.99 & 0.93 & 0.73 & 0.66 & 0.95 & 0.65 & 0.96 & 0.66 & 0.61 \\
\hline $\begin{array}{l}\text { ToxinPred } \\
\text { server* }\end{array}$ & $M L$ & 0.55 & 0.98 & $N / A$ & $N / A$ & 0.55 & 0.98 & 0.82 & 0.93 & 0.66 & 0.63 \\
\hline
\end{tabular}




\section{Table 3 (on next page)}

Comparison of classification performance for calibrated ToxClassifier to BLAST based annotation models and ClanTox toxin prediction server 
1 Table 3: ToxClassifier calibrated meta-classifier, Test set results compared to oneBLAST, naiveBLAST and ClanTox annotation 2 methods

3

\begin{tabular}{l|c|c|c|c|c|c|c|c}
\hline \multicolumn{6}{c}{ Classification performance of ToxClassifier meta-classifier, compared to BLAST based methods and ClanTox server } \\
\hline \hline Annotation Model & Tool & Accuracy & $\begin{array}{c}\text { Positive } \\
\text { Prediction } \\
\text { Value }\end{array}$ & $\begin{array}{c}\text { Negative } \\
\text { Prediction } \\
\text { Value }\end{array}$ & Sensitivity & Specificity & F1-value \\
\hline naiveBLAST & BLAST & 0.976 & 0.294 & 0.998 & 0.857 & 0.977 & 0.438 & 0.494 \\
\hline oneBLAST & BLAST & 0.994 & 0.660 & 1.000 & 0.951 & 0.995 & 0.779 & 0.790 \\
\hline ClanTox server & ML-based & 0.947 & 0.209 & 1.000 & 0.954 & 0.976 & 0.342 & 0.440 \\
\hline ToxClassifier & ML-based & 0.997 & 0.825 & 1.000 & 0.967 & 0.998 & 0.890 & 0.892 \\
\hline
\end{tabular}

4 


\section{Table 4 (on next page)}

Results of expert manual annotation to ToxClassifier annotation for set of novel proteins from venom gland transcriptome of Bothrops atrox snake. 
1 Table 4: Blinded 'ToxClassifier' prediction of proteins as toxins or non-toxins from the standard 2 annotation of the venom gland transcriptome of Bothrops atrox. 3

\begin{tabular}{l|l|l}
\hline \multicolumn{2}{c}{ Sequence annotation } \\
\hline \hline Test Sample & 'ToxClassifier' & Standard annotation \\
\hline Test 01 & Potential toxin & BPP precursor - Toxin \\
\hline Test 02 & Potential toxin & Crisp - Toxin \\
\hline Test 03 & Toxin & CTL - Toxin \\
\hline Test 04 & Toxin & CTL - Toxin \\
\hline Test 05 & Potential toxin & Hyaluronidase - Toxin \\
\hline Test 06 & Potential toxin & Kunitz -like sequence - Probably a toxin \\
\hline Test 07 & Toxin & LAAO - Toxin \\
\hline Test 08 & Not a toxin & Nucleotidase - Probably not a toxin \\
\hline Test 09 & Not a toxin & Phosphodiesterase - Probably not a toxin \\
\hline Test 10 & Toxin & PLA2 - Toxin \\
\hline Test 11 & Toxin & SVMP class PI - Toxin \\
\hline Test 12 & Toxin & SVMP class PII - Toxin \\
\hline Test 13 & Toxin & SVMP class PIII - Toxin \\
\hline Test 14 & Toxin & SVSP - Toxin \\
\hline Test 15 & Potential toxin & VEGF - Toxin \\
\hline
\end{tabular}

